\title{
Resource Allocation Techniques for Heterogeneous Networks Under User Misbehavior
}

\author{
Vasileios Miliotis, Member, IEEE, Luis Alonso, Senior Member, IEEE, \\ and Christos Verikoukis, Senior Member, IEEE
}

\begin{abstract}
In this letter we focus on the uplink offloading with IP Flow Mobility (IFOM). With IFOM a User Equipment (UE) is able to maintain concurrently two data streams, one through LTE and the other through WiFi. We consider the existence of malicious UEs that aim to exploit the WiFi bandwidth against their truthful peers, in order to upload less data through the energy demanding LTE uplink and a reputation based method is proposed to combat the selfish operation. The WiFi bandwidth is allocated based on weighted proportional fairness and the LTE rate is defined through an exponential pricing algorithm. We theoretically analyse our approach and evaluate the performance of the malicious and the truthful UEs in terms of energy efficiency and throughput, through simulations. We show that while the malicious UEs present better energy efficiency before being detected, their performance is significantly degraded with the proposed reaction method.
\end{abstract}

Index Terms-Resource allocation, Heterogeneous networks, Misbehavior.

\section{INTRODUCTION}

$\mathbf{T}$ HE relatively low deployment cost of WiFi Access Points (APs) has led the providers and the research community to investigate offloading techniques for the cellular networks through WiFi. With the release-10 of 3GPP, a UE in LTE networks is able to concurrently maintain connections with the cellular network and a WiFi AP, in order to offload part of its traffic. The scheme that allows this connectivity is named IP Flow Mobility (IFOM) [1]. This technology allows an operator or a UE to shift an IP flow to a different radio access technology. A UE may shift a file upload on the WiFi network and, when it moves out of the AP coverage, it will make a seamless shift back to the cellular network. Another case is the division of a UE's data flow into two sub-flows and the service of each sub-flow by different radio access technologies, as proposed in [2]. Previous offloading research has focused on the downlink traffic offloading and do not consider the increasing tendency of uploading user created content. In [3], methods for session continuity are proposed for non-seamless WiFi offloading in LTE networks. Taking into consideration the shared nature of WiFi resources, the access algorithms for uplink offloading need to be fair. Namely, the UEs must be truthful when declaring their uplink data needs. In [4] and [5] selfish detection mechanisms are proposed for WLAN and WiFi tethering respectively.

In this letter we assume the existence of malicious UEs that try to exploit resources against their peers and we propose

Manuscript received September 9, 2015. The associate editor coordinating the review of this letter and approving it for publication was D. W. Kwan Ng.

V. Miliotis (corresponding author, vasileios.miliotis@tsc.upc.edu) and L. Alonso (luisg@tsc.upc.edu) are with the Dept. of Signal Theory and Communications, Polytechnic University of Catalonia (UPC), Barcelona, Spain.

C. Verikoukis (cveri@cttc.es) is with the Telecommunications Technological Centre of Catalonia (CTTC), Castelldefels, Spain.

This work was supported by the Research Projects 2014-SGR-1160, 2014 SGR-1551, CellFive (TEC2014-60130-P), DEFINE-5G (TEC2014-60258-C2 2-R). a reaction method to combat this malicious operation. We focus on the uplink traffic offloading with IFOM and its impact on the energy efficiency and throughput of modern mobile communication devices. We propose a reputation based technique and we evaluate the performance of malicious and truthful UEs, before and after the misbehavior detection. The WiFi access is based on weighted proportional fairness which is affected by the malicious operation and the LTE access on exponential pricing, for which we provide a detailed mathematical analysis. In [6], the same system model was studied without malicious UEs and in [7], a mathematical proof was provided for the weighted proportionally fair WiFi bandwidth allocation.

\section{System MODEL}

We consider an LTE eNodeB with its coverage area partially covered by several WiFi APs of the same LTE provider. We focus on $N$ LTE UEs that are under the concurrent coverage of the eNodeB and one of the deployed APs. All UEs are equipped with WiFi network interface, in addition to their LTE connectivity, and conform to IFOM. An IP flow can be divided into two sub-flows and the UEs can define their size. One sub-flow is directed through LTE and the other through WiFi. Each $\mathrm{UE}_{i}$ needs to upload a file of size equal to $K_{i}$, where $i=(1, \ldots, N) . K_{i}$ takes values between $K_{\text {min }}$ and $K_{\max }$. When a UE wants to upload a file, it informs the eNodeB for its data needs and this information is disseminated to the corresponding AP for the WiFi bandwidth allocation. The described scheme is applied to each one of the WiFi APs and we investigate the uplink data offloading for time horizons of duration equal to $\Delta T$.

We consider the existence of $L$ untruthful UEs that declare upload needs equal to $K_{\max }$, which is more than their real needs, aiming to gain more WiFi bandwidth to offload, while the rest of the UEs are truthful. The WiFi bandwidth is allocated based on weighted proportional fairness, and the upload needs of the users are part of the weighting factor. Hence, the more the declared upload data needs, the more WiFi bandwidth is allocated to a UE. After the end of each offloading period, the LTE Evolved Packet Core (EPC), where the packet filtering operation is being done, is able to identify a malicious operation and inform the WiFi AP for future allocation. We define a reputation vector $\mathbf{v}_{j}$, with $\mathrm{v}_{j}(i) \in(0,1]$ to represent the truthfulness of the UEs during the $j^{\text {th }}$ offloading period of duration equal to $\Delta T$. At the start, every $\mathrm{UE}_{i}$ is considered truthful and its reputation value is equal to $\mathrm{v}_{1}(i)=1$. After an offloading period $j$, the reputation vector is updated as follows

$$
\begin{aligned}
& \mathbf{v}_{j+1}(i)=1 \text {, if } \mathrm{UE}_{i} \text { truthful in } j^{t h} \text { period } \\
& \mathbf{v}_{j+m}(i)=K_{i} / K_{\text {max }} \text {, if } \mathrm{UE}_{i} \text { untruthful in } j^{\text {th }} \text { period }
\end{aligned}
$$

Where $m=1, \ldots, M$. A $\mathrm{UE}_{i}$ that is untruthful during the offloading period $j$ is punished according to (1) for the following 
$M$ offloading periods. After these periods, the reputation of the untruthful $\mathrm{UE}_{i}$ is reset to $\mathrm{v}_{j+M+1}(i)=1$. Truthful UEs maintain their reputation value equal to one. Each $\mathrm{UE}_{i}$ offloads part of its data needs $K_{i}$ and the rest is uploaded through its LTE connection. We assume that the channel characteristics between each $\mathrm{UE}_{i}$ and the LTE eNodeB are described by a normalized spectrum efficiency $\theta_{i} \in[0,1]$, such that for a bandwidth allocation that gives to $\mathrm{UE}_{i}$ the ability to upload with an uplink rate equal to $R_{i}^{L T E}$, the actual achieved uplink rate is equal to $\theta_{i} R_{i}^{L T E}$.

\section{A. LTE Uplink Power Model}

Regarding the LTE uplink power level of the UEs, we adopt the energy model proposed by Huang et al. in [8]. According to this model the power level of the $\mathrm{UE}_{i}$ 's LTE interface during uplink transmission is expressed as

$$
P_{i}^{L T E}=\alpha_{u} R_{i}^{L T E}+\beta[\mathrm{mW}]
$$

where $\alpha_{u}$ is the uplink transmission power per Mbps, $R_{i}^{L T E}$ is the LTE uplink rate of $\mathrm{UE}_{i}$ (in Mbps) and $\beta$ is the base power of the LTE card.

\section{B. IEEE 802.11 DCF Energy Consumption in the Uplink}

Following Bianchi's analysis [9] for saturated traffic conditions and ideal channel conditions, we notice that the throughput of a user that tries to upload data through WiFi is significantly affected by the number of users that are under the coverage of the same AP. The per user uplink throughput is denoted by $S(N)$ (in Mbps), where $N$ is the number of contending users. A user's energy efficiency $E E(N)$ (in bits/Joule), is also a function of the number of contending users $N$ and we assume that the WiFi interface operates with power levels denoted by $P_{T x}^{W i F i}, P_{R x}^{W i F i}$ and $P_{i d l e}^{W i F i}$ for transmit, receive and idle modes respectively.

\section{Uplink Offloading Energy Consumption}

Every UE under the concurrent coverage of the two access technologies will have the opportunity to offload $w_{i} K_{i}$ bits through the WiFi AP, where $w_{i} \in[0,1]$ for $i=(1, \ldots, N)$. The remainder data volume $\left(1-w_{i}\right) K_{i}$ is transmitted through the LTE connection of each UE. Every $\mathrm{UE}_{i}$ with data needs equal to $K_{i}$ that offloads its uplink according to $w_{i}$ will present energy consumption $E C_{i}(N)$ as a function of the number of contending UEs $N$, which is expressed as

$$
E C_{i}(N)=\left(1-w_{i}\right) K_{i} \frac{P_{i}^{L T E}}{R_{i}^{L T E}}+w_{i} K_{i} \frac{1}{E E(N)} \text { [Joule] }
$$

\section{Weighted Proportionally Fair WiFi ACCESS}

The UEs offload part of their data needs through the WiFi according to the Proportionally Fair Bandwidth (PFB) allocation algorithm that we hereby analyse. Each $\mathrm{UE}_{i}$ is allocated bandwidth equal to $r_{i}, i=(1, \ldots, N)$, such that $\sum_{i=1}^{N} r_{i} \leq R_{i}^{W i F i}$. The allocation during the offloading period $j$ is proportionally fair over the ratio $\rho_{i}=\mathrm{v}_{j}(i) K_{i} / \theta_{i}$, where $\mathrm{v}_{j}(i)$ is the reputation value that corresponds to $\mathrm{UE}_{i}$. The proportionally fair allocation is represented by a rate allocation vector $\mathbf{r}=\left(r_{1}, \ldots, r_{N}\right)$ for $\mathbf{r} \geq 0$ and $\sum_{i=1}^{N} r_{i} \leq R_{i}^{W i F i}$, where:

$$
r_{i}=\frac{\rho_{i}}{\sum_{i=1}^{N} \rho_{i}} R^{W i F i}
$$

We aim to allocate exclusive access periods to each $\mathrm{UE}_{i}$ equal to $t_{i}$, for $i=(1, \ldots, N)$. In these periods the UEs will be able to transmit through the WiFi AP with throughput $R^{W i F i}=S(1)$. We transform the proportionally fair bandwidth allocation into proportionally fair airtime allocation by having $r_{i} \Delta T=t_{i} S(1)$. Hence, the weighted proportionally fair airtime allocation is equal to

$$
t_{i}=\frac{\rho_{i}}{\sum_{i=1}^{N} \rho_{i}} \Delta T
$$

Regarding the implementation of the PFB algorithm we adopt the idea of unsolicited Clear To Send (CTS) frames initiated by the AP that was proposed in [10].

\section{LTE PRICING SCHEME}

The LTE uplink power of a $\mathrm{UE}_{i}$, following the power model of (2), is a function of its LTE uplink transmission rate, $R_{i}^{L T E}$. Following, we propose a two-stage LTE pricing scheme, where the LTE operator in the first step decides the price $p$ per unit of transmit rate and in the second step the UEs decide the rate for which they intend to pay as a function of the price and the spectrum efficiency they experience. We approach the pricing problem using backward induction, examining first the UEs' demands (Stage II) and then the operator's decision on the price (Stage I).

Stage II: The payoff function of a $\mathrm{UE}_{i}$, for acquiring $R_{i}^{L T E}$ quantity of uplink rate, with a price $p$ per unit of rate, following the exponential pricing model, is expressed as

$$
U_{i}^{\exp }\left(R_{i}^{L T E}\right)=\ln \left(1+\theta_{i} R_{i}^{L T E}\right)-p\left(e^{R_{i}^{L T E}}-1\right)
$$

This payoff function of a $\mathrm{UE}_{i}$ under exponential pricing, with normalized spectrum efficiency $\theta_{i}$, is equal to the logarithmic utility function, that expresses the diminishing return of getting additional resources, minus the exponential price that the $\mathrm{UE}_{i}$ has to pay for acquiring $R_{i}^{L T E}$ quantity of rate. We notice that $U_{i}^{e x p}\left(R_{i}^{L T E}\right)$ is a concave function, since $U_{i}^{e x p}\left(R_{i}^{L T E}\right)^{\prime \prime}=$ $-\left(\theta_{i} /\left(1+\theta_{i} R_{i}^{L T E}\right)\right)^{2}-p e^{R_{i}^{L T E}}<0$. Thus, it has only one maximum, and therefore the local maximum is also the global maximum. Differentiating (6) we have

$$
\frac{\partial U_{i}^{e x p}}{\partial R_{i}^{L T E}}=\frac{\theta_{i}}{1+\theta_{i} R_{i}^{L T E}}-p e^{R_{i}^{L T E}}=0
$$

We need to solve this non-linear equation with respect to $R_{i}^{L T E}$. (7) can be rewritten as

$$
\ln \left(\frac{1}{p}\right)+\frac{1}{\theta_{i}}=\left(R_{i}^{L T E}+\frac{1}{\theta_{i}}\right)+\ln \left(R_{i}^{L T E}+\frac{1}{\theta_{i}}\right)
$$

For $x=R_{i}^{L T E}+\frac{1}{\theta_{i}}$ and $y=\ln \left(\frac{1}{p}\right)+\frac{1}{\theta_{i}}$, (8) becomes

$$
y=x+\ln x
$$

which after some straight forward mathematical manipulations can be written as

$$
x e^{x}=e^{y}
$$

Taking the value of the Lambert $\mathrm{W}$ function [11] of each part of (10) and using the Lambert $\mathrm{W}$ function identity $W\left(x e^{x}\right)=$ $x$ we have $x=W\left(e^{y}\right)$. Replacing $x$ and $y$ we have

$$
R_{i}^{L T E}=W\left(\frac{e^{\frac{1}{\theta_{i}}}}{p}\right)-\frac{1}{\theta_{i}}
$$

Stage I: The price $p$ that the provider decides in the exponential pricing model is such, that the UE with the $\max \left(\theta_{i}\right)$ is allocated the maximum value of the LTE uplink 
rate $R_{\max }^{L T E}$. The price is formed according to (12).

$$
p=\frac{\max \left(\theta_{i}\right)}{\left(1+\max \left(\theta_{i}\right) R_{\max }^{L T E}\right) e^{R_{\max }^{L T E}}}, i=(1, \ldots, N)
$$

In order for a $\mathrm{UE}_{i}$ under the coverage of the $\mathrm{AP}$ to have the opportunity to upload part of its data needs through the eNodeB, its allocated uplink LTE rate has to be positive. Namely, the following condition needs to stand:

$$
W\left(\frac{e^{\frac{1}{\theta_{i}}}}{p}\right)-\frac{1}{\theta_{i}}>0
$$

Otherwise, $\mathrm{UE}_{i}$ will only upload through the WiFi AP. The allocated rate to each $\mathrm{UE}_{i}$, that satisfies (13), following the exponential pricing model is expressed as

$$
R_{i}^{L T E}=W\left(\frac{\left(1+\max \left(\theta_{i}\right) R_{\max }^{L T E}\right) e^{R_{\max }^{L T E}+\frac{1}{\theta_{i}}}}{\max \left(\theta_{i}\right)}\right)-\frac{1}{\theta_{i}}
$$

Following we provide analytical expressions for the average energy consumption of the offloading UEs for both truthful and malicious UEs.

A. Energy Efficiency of the Truthful UEs

The average per truthful UE energy consumption of the WiFi network interface, while uploading, is expressed as

$$
\overline{E C}_{T x}^{W i F i}=\frac{1}{N-L}\left(\sum_{i=1}^{N-L} \frac{K_{i} / \theta_{i}}{\sum_{i=1}^{N} \rho_{i}} \Delta T \frac{S(1)}{E E(1)}\right) \text { [Joule] }
$$

The average per UE energy consumption of the LTE network interface card is equal to

$$
\overline{E C}^{L T E}=\frac{1}{N-L} \sum_{i=1}^{N-L}\left(\left(K_{i}-t_{i} S(1)\right) \frac{P_{i}^{L T E}}{\theta_{i} R_{i}^{L T E}}\right) \text { [Joule] }
$$

Combining (15) and (16) the average per UE energy efficiency of IFOM offloading under the PFB algorithm is expressed in (17).

$$
\bar{E}_{\text {eff }}^{P F B}=\frac{\frac{1}{N-L} \sum_{i=1}^{N-L} K_{i}}{\overline{E C}_{T x}^{W i F i}+\overline{E C}^{L T E}} \text { [bits/Joule] }
$$

\section{B. Energy Efficiency of the Malicious UEs}

The average per malicious UE energy consumption of its WiFi network interface, during the uploading phase, is expressed as

$$
\overline{E C}_{T x, m a l}^{W i F i}=\frac{1}{L} \sum_{i=1}^{L}\left(\frac{\mathrm{v}_{j}(i) K_{i} / \theta_{i}}{\sum_{i=1}^{N} \rho_{i}} \Delta T \frac{S(1)}{E E(1)}\right) \quad \text { [Joule] }
$$

The malicious UE's energy consumption of the LTE network interface card is equal to

$$
\overline{E C}_{\text {mal }}^{L T E}=\frac{1}{L} \sum_{i=1}^{L}\left(\left(K_{i}-t_{i} S(1)\right) \frac{P_{i}^{L T E}}{\theta_{i} R_{i}^{L T E}}\right) \text { [Joule] }
$$

Combining (18) and (19) the malicious UEs' energy efficiency of IFOM uplink offloading under the PFB algorithm is

$$
\bar{E}_{\text {eff }, \text { mal }}^{P F B}=\frac{\frac{1}{L} \sum_{i=1}^{L} K_{i}}{\overline{E C}_{T x, m a l}^{W i F i}+\overline{E C}_{m a l}^{L T E}}[\text { bits/Joule] }
$$

\section{NumeriCAL RESUlts}

We evaluate the system under consideration by running extensive simulations using MATLAB ${ }^{\mathrm{TM}}$. We present the performance of malicious UEs in comparison to truthful UEs which are situated under the concurrent coverage of the same eNodeB and WiFi AP for 10 offloading UEs in total. We examine the system performance under the existence of one to ten malicious UEs. We compare the performance of the malicious to truthful UEs, in terms of energy efficiency and throughput, before and after the update of the reputation vector. The simulations are repetitively conducted for offloading time periods of $\Delta T=5 \mathrm{sec}$, chosen arbitrarily, and for $M=1,2, \ldots, 10$ consecutive punishment periods. For $M \geq 3$ there is no incentive for a malicious UE to be untruthful from the energy efficiency perspective. We present our results for $M=7$ consecutive punishment periods to represent the policy of a strict provider towards malicious operation. The data volume needs of the UEs are assumed to follow a uniform distribution of file sizes between 5-15 MB. These data needs represent the volume of a photo to a small video, created by contemporary smartphones. The UEs that operate in malicious mode declare their data volume equal to $K_{\max }=15 \mathrm{MB}$. The uplink power level of $\mathrm{UE}_{i}$ 's LTE interface card, $P_{i}^{L T E}$, is assumed to follow (2) as a function of its LTE uplink rate, which is defined by the exponential pricing algorithm. We perform the simulations for $\theta_{i} \in[0.8,1]$. The IEEE 802.11 network interface card power levels $P_{T x}^{W i F i}, P_{R x}^{W i F i}$ and $P_{i d l e}^{W i F i}$ are assumed to follow the measurements provided in [12]. The numerical values of the simulation parameters are presented in Table I.

In Fig. 1(a) we present the energy efficiency results for one to ten malicious UEs and for spectrum efficiency $\theta_{i} \in[0.8,1]$ and we can see that analysis and simulations perfectly fit. It is notable that the malicious UEs perform better compared to the average energy consumption of truthful UEs ranging from $15.5 \%$ for one malicious UEs to $8.4 \%$ for nine malicious UEs. This happens because the malicious UEs, by declaring more uplink data volume needs than their real, more WiFi bandwidth is allocated to them and they upload less data through their LTE connections, which are more energy consuming. In Fig. 1(b), we can see that after the update of the reputation vector the energy efficiency of the malicious UEs is deteriorated as the punishment rule applies. This deterioration varies from $7.5 \%$ for eight UEs to $19 \%$ for one to nine malicious UEs compared to truthful UEs. We also notice that the truthful UEs present better performance for larger number of punished

TABLE I

Simulation PARAMETERS

\begin{tabular}{ll}
\hline Parameter & Value \\
\hline LTE max uplink rate $R_{\max }^{L T E}$ & $5 \mathrm{Mbps}$ \\
LTE uplink power per Mbps $\alpha_{u}$ & $438.39 \mathrm{~mW} / \mathrm{Mbps}$ \\
LTE base power $\beta$ & $1288.04 \mathrm{~mW}$ \\
WiFi packet payload & $1500 \mathrm{bytes}$ \\
WiFi Data $\left(R^{W i F i}\right) /$ Ctrl. transmission rate & $54 / 6 \mathrm{Mbps}$ \\
$P_{T x}^{W i F i} / P_{R x}^{W i F i} / P_{i d l e}^{W i F i}$ & $1900 / 1340 / 1340 \mathrm{~mW}$ \\
SIFS/ DIFS & $10 / 50 \mu \mathrm{sec}$ \\
\hline
\end{tabular}




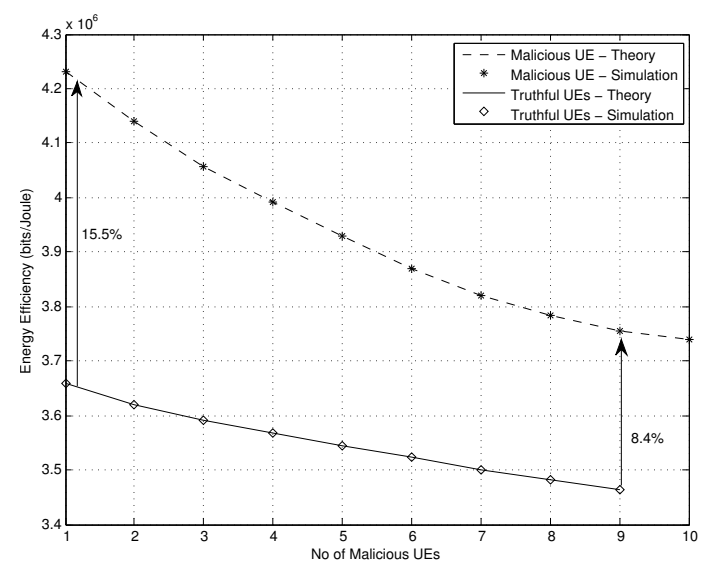

(a) During malicious operation.

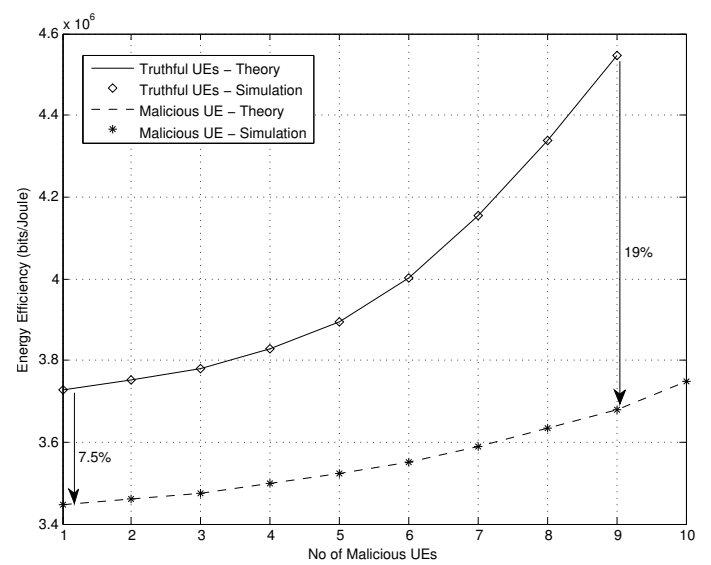

(b) After reputation vector update.

Fig. 1. Energy efficiency for different number of malicious UEs.

UEs. This happens because the WiFi bandwidth that is not allocated to the malicious UEs is proportionally allocated to the truthful UEs, helping them upload less data through their LTE connections. In Fig. 2(a), we present an energy efficiency comparison for long term system performance, for the cases of one to nine UEs. We notice that a malicious UE presents from $4.7 \%$ to $16.4 \%$ less energy efficiency compared to a truthful UE. In Fig. 2(b), we present a throughput comparison for long term system performance for the case of a truthful and nine malicious UEs, assuming they experience the same channel conditions $\theta_{i} \in[0.8,0.1]$ with a step equal to 0.02 . We notice that in long term a malicious UE presents from $14.1 \%$ to $11.9 \%$ less throughput compared to a truthful UE.

\section{CONCLUSION}

In this letter we focus on the uplink offloading with IFOM, with the existence of malicious UEs where the WiFi access is based on weighted proportional fairness and the LTE rate allocation on exponential pricing. We propose a reputation based reaction method to combat the malicious operation and we present comparison results on the energy efficiency and throughput of the malicious and the truthful UEs.

\section{REFERENCES}

[1] C. Sankaran, "Data Offloading Techniques in 3GPP Rel-10 Networks: A tutorial," IEEE Commun. Mag., vol. 50, no. 6, pp. 46-53, June 2012.

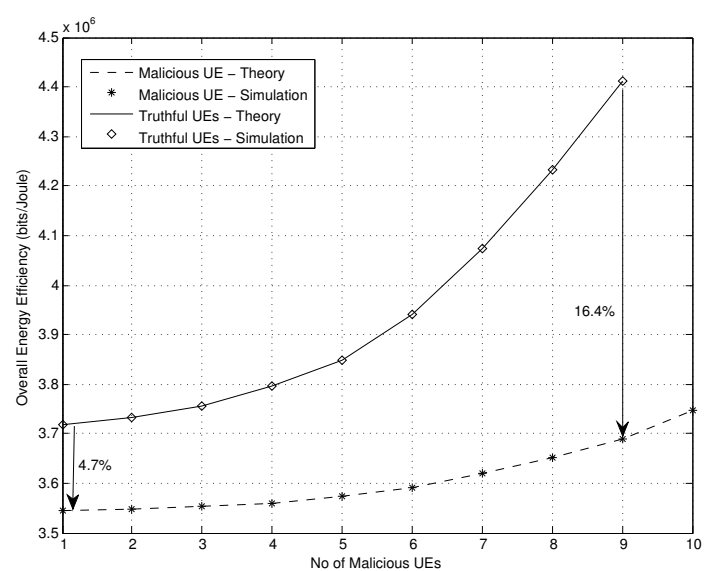

(a) Energy efficiency comparison.

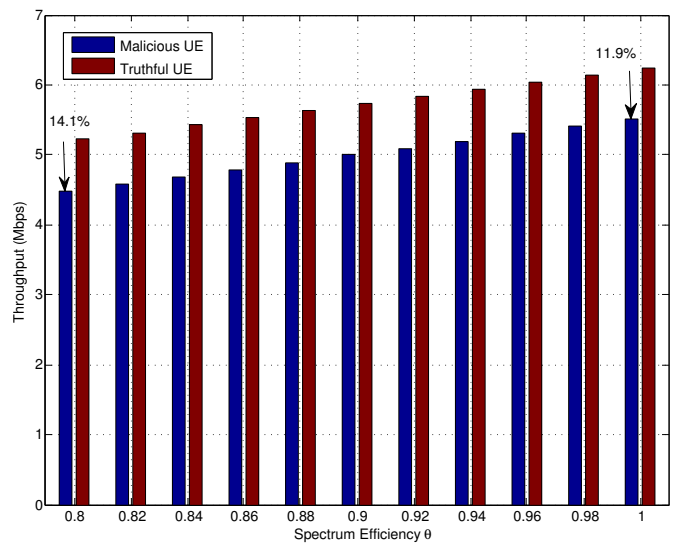

(b) Throughput comparison.

Fig. 2. Long term system performance for $\theta_{i} \in[0.8,1]$.

[2] H. Son, S. Lee, S.-C. Kim, and Y.-S. Shin, "Soft Load Balancing Over Heterogeneous Wireless Networks," IEEE Transactions on Vehicular Technology, vol. 57, no. 4, pp. 2632-2638, July 2008.

[3] W. Yoon and B. Jang, "Enhanced Non-Seamless Offload for LTE and WLAN Networks," IEEE Commun. Lett., vol. 17, no. 10, pp. 1960-1963, Oct. 2013.

[4] P. Serrano, A. Banchs, V. Targon, and J. Kukielka, "Detecting Selfish Configurations in 802.11 WLANs," IEEE Commun. Lett., vol. 14, no. 2, pp. 142-144, 2010.

[5] J. Choi, A. W. Min, and K. G. Shin, "On Selfish Configuration in Wi-Fi Tethering," IEEE Commun. Lett., vol. 17, no. 5, pp. 841-843, 2013.

[6] V. Miliotis, L. Alonso, and C. Verikoukis, "Offloading with IFOM: The Uplink Case," in IEEE Global Communications Conference (GLOBECOM). IEEE, 2014, pp. 2661-2666.

[7] — , "Energy Efficient Proportionally Fair Uplink Offloading for IP Flow Mobility," in IEEE 19th International Workshop on Computer Aided Modeling and Design of Communication Links and Networks (CAMAD). IEEE, 2014, pp. 6-10.

[8] J. Huang, F. Qian, A. Gerber, Z. M. Mao, S. Sen, and O. Spatscheck, "A Close Examination of performance and Power Characteristics of $4 \mathrm{G}$ LTE Networks," in Proc. of the 10th ACM International Conference on Mobile Systems, Applications, and Services, June 2012.

[9] G. Bianchi, "Performance Analysis of the ieee 802.11 Distributed Coordination Function," IEEE J. Sel. Areas Commun, vol. 18, no. 3, pp. 535-547, Mar. 2000.

[10] Y. Li, D. Papagiannaki, and A. Sheth, "Uplink Traffic Control in Home 802.11 Wireless Networks," in Proc. of the 2nd ACM SIGCOMM Workshop on Home Networks, Aug. 2011.

[11] R. M. Corless, G. H. Gonnet, D. E. Hare, D. J. Jeffrey, and D. E. Knuth, "On the Lambert W Function," Advances in Computational Mathematics, vol. 5, no. 1, pp. 329-359, 1996.

[12] J. Ebert, S. Aier, G. Kofahl, A. Becker, B. Burns, and A. Wolisz, "Measurement and Simulation of the Energy Consumption of an WLAN Interface," Technical University of Berlin, Telecommunication Networks Group, Tech. Rep. TKN-02-010, June 2002. 